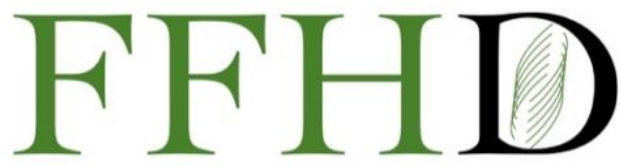

Functional Foods in Health and Disease

\title{
Black tea decreases postprandial blood glucose levels in healthy humans and contains high-molecular-weight polyphenols that inhibit $\alpha$-glucosidase and $\alpha$-amylase in vitro: a randomized, double blind, placebo-controlled, crossover trial
}

\author{
Yoshikazu Isono ${ }^{1, *}$, Hisako Watanabe ${ }^{1}$, Masafumi Kumada ${ }^{1}$, Tsuyoshi Takara ${ }^{2}$, and \\ Shin-ichro lio ${ }^{3}$
}

\begin{abstract}
${ }^{1}$ Biwako Research Institute, Otsuka Foods Co., Ltd., Otsu, Shiga, Japan. ${ }^{2}$ Medical Corporation Seishinkai, Takara Clinic, Shinagawa-ku, Tokyo, Japan. ${ }^{3}$ ORTHOMEDICO Inc., Bunkyo-ku, Tokyo, Japan.
\end{abstract}

*Corresponding author: Yoshikazu Isono, PhD, Biwako Research Institute, Otsuka Food Co., Ltd., 1-11-1, Karasaki, Otsu, Shiga, 520-0106, Japan.

Submission Date: March 16 ${ }^{\text {th }}$ 2021; Acceptance Date: April 30 ${ }^{\text {th }}, 2021$; Publication Date: May $11^{\text {th }}, 2021$

Please cite this as: Isono Y., Watanabe H., Kumada M., Takara T., lio S. Black tea decreases postprandial blood glucose levels in healthy humans and contains high-molecular-weight polyphenols that inhibit $\alpha$-glucosidase and $\alpha$-amylase in vitro: a randomized, double blind, placebo-controlled, crossover trial. Functional Foods in Health and Disease 2021; 11(5): 222-237. DOI: https://www.doi.org/10.31989/ffhd.v11i5.791

\footnotetext{
ABSTRACT

Background: To prevent diabetes, it is important to control postprandial glycemic levels. Studies have suggested that consuming black tea decreases the risk of type 2 diabetes; however, only a few studies have examined the effects of black tea on postprandial glycemic control after consuming starch-rich foods. In addition, the mechanism underlying the suppression of postprandial glucose levels remains unclear.

Objective: To investigate the effects of black tea on postprandial blood glucose levels in healthy humans and to identify the components of black tea that inhibit digestive enzymes.
} 


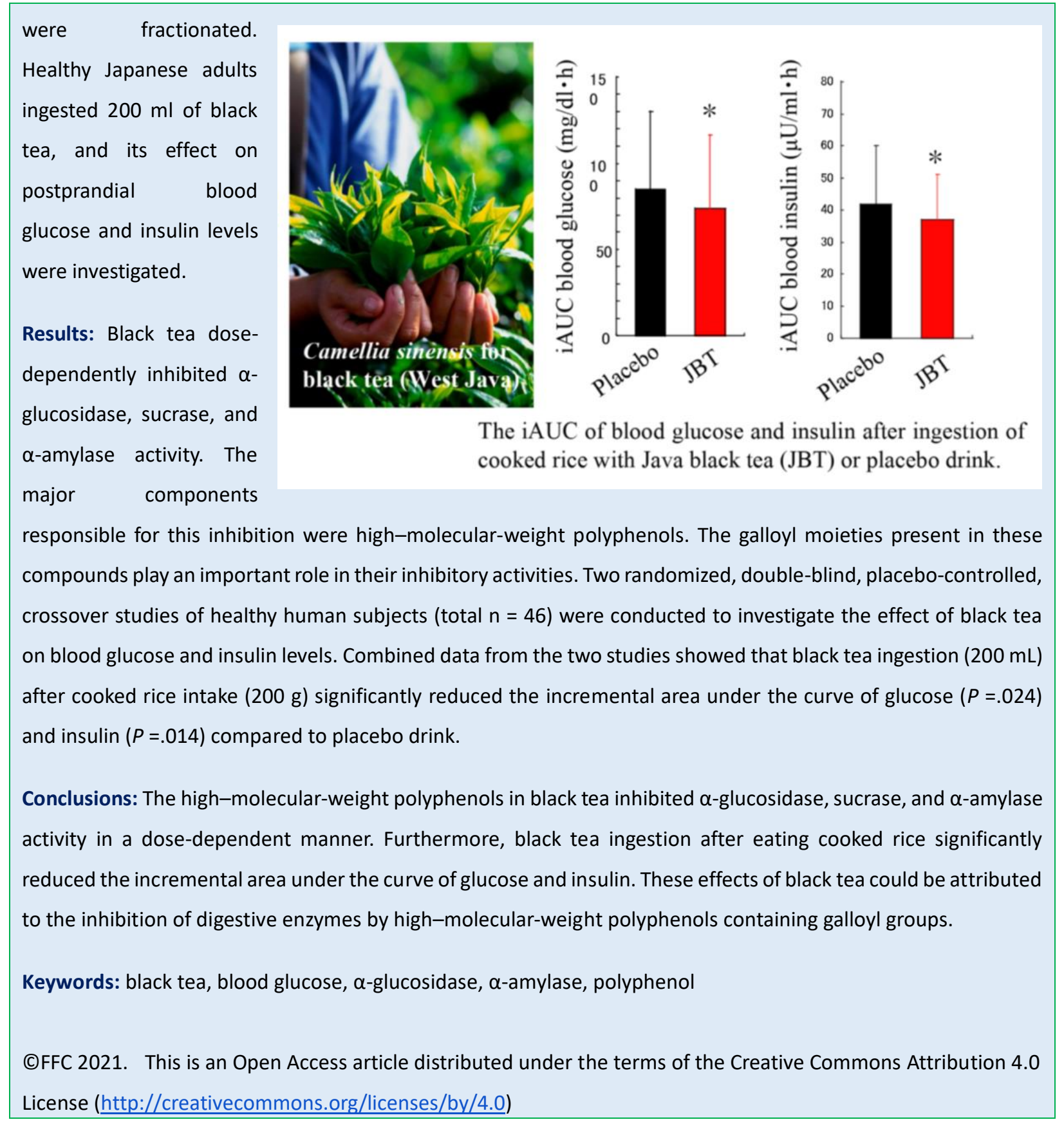

\section{INTRODUCTION}

According to the International Diabetes Federation (IDF), 463 million people worldwide had diabetes in 2019, an increase of 38 million from 2017 [1]. The prevalence of diabetes in adults is presently $9.6 \%$. Without effective preventive measures, the number of affected individuals is projected to increase to 578 million by 2030 and 700 million by 2045 . Worldwide, the cost of diabetes-related healthcare in 2019 was about 760 billion dollars and the healthcare burden of diabetes is increasing. Alternative treatments can be used as a complement to conventional medicine to prevent and treat diabetes. Functional foods and nutritional supplements are promising alternative treatments for the regulation of plasma glucose to 
prevent diabetes, thereby avoiding the cost of treating diabetes and its complications.

Type 2 diabetes, accounting for $90 \%$ of all cases, is promoted by social, economic, environmental, and genetic factors that include urbanization, aging, lack of exercise, overeating, and overweight and obesity [1]. Postprandial glycemic control is important for preventing diabetes and delaying its complications [2, 3]. Postprandial plasma glucose levels are determined by the amount and digestion rate of consumed carbohydrates. Therefore, one therapeutic approach to controlling postprandial hyperglycemia is to inhibit digestion and delay the subsequent absorption of dietary carbohydrates [4]. Dietary carbohydrates are digested by $\alpha$-amylase in the small intestine to generate oligosaccharides and disaccharides, which are then hydrolyzed by $\alpha$-glucosidase to produce individual monosaccharides. Inhibiting $\alpha$-amylase and $\alpha$ glucosidase is a strategy for managing type 2 diabetes because the resulting decrease in glucose generation effectively suppresses postprandial hyperglycemia [5].

$\alpha$-Amylase and $\alpha$-glucosidase inhibitors are present in a variety of plants [6]. Plant polyphenols have been shown to inhibit $\alpha$-amylase and $\alpha$-glucosidase in vitro $[7,8,9]$. Black tea, made from the fresh leaves of Camellia sinensis, is one of the most widely consumed beverages in the world. During the fermentation process, polyphenols, such as catechins in tea leaves, are converted to polymerized polyphenols, including theaflavins and thearubigins [10]. These polymerized polyphenols are reported to slow sugar absorption by inhibiting $\alpha$-amylase and $\alpha$-glucosidase in vitro and in vivo $[11,12,13,14]$, with theaflavins exhibiting the strongest inhibitory effects $[12,13]$. Theaflavin is reported to be a major source of $\alpha$-glucosidase inhibition among black tea polyphenols [15]. Kusano et al.[16] report that polymer-like oxidation products are more important than theaflavins as inhibitors of pancreatic lipase and amylase in black tea extract.

Evidence suggests that black tea consumption decreases the risk of type 2 diabetes. Traditional medical practice in Sri Lanka recommends that diabetic patients be given 6-10 cups of tea [17]. In Singapore, those who drink one or more cups of black tea daily have a $14 \%$ lower risk of diabetes than do non-teadrinkers [18]. Zhong et al. [19] found that a mixture of black, green, and mulberry tea extracts induced malabsorption of $25 \%$ of ingested carbohydrates in healthy adults. Another study showed that ingestion of $1 \mathrm{~g}$ of instant black tea reduced the late phase blood glucose response in healthy humans, with a corresponding increase in insulin after glucose consumption [20]. Butacnum et al. [21] reported that black tea containing polymerized polyphenol significantly decreased the incremental blood glucose area under the curve (AUC) after sucrose intake, with no significant difference in insulin levels. However, few clinical studies have investigated the effects of black tea on postprandial glycemic control in humans after consuming starch-rich foods. In addition, the mechanism underlying the suppression of postprandial glucose levels remains unclear. This study aims to investigate the effects of black tea on plasma glucose levels in healthy humans after eating cooked rice and to 
identify the components of black tea that inhibit digestive enzymes.

\section{MATERIAL AND METHODS}

Black tea and reagents: Java black tea (JBT) with no sugar added was obtained from Otsuka Foods Co. Ltd. This product is an extract of black tea leaves harvested in West Java, Indonesia. Salacia extract containing $0.13 \%$ of salacinol was obtained from Pearl Ace Corp. (Tokyo). $\alpha$-Amylase from porcine pancreas (700-1400 Units/mg protein) and rat intestinal acetone powder were purchased from Sigma-Aldrich Co. (St. Louis, MO, USA). Tannase was obtained from Mitsubishi Chemical Foods Co. (Tokyo, Japan). Polystyrene standards were purchased from Tosoh Co. (Tokyo, Japan). All other chemicals used in the experiments were of the highest purity commercially available.

Analysis of JBT polyphenol content: The polyphenol content of JBT was analyzed using an HPLC system comprising a SIL-20ACXR autosampler, CBM-20A communication bus model, CTO-20AC column oven, SPD-20A UV-VIS detector, and LC-20ADXR reciprocating pumps (Shimadzu Co., Kyoto, Japan) equipped with a Sun Fire C18 column $250 \mathrm{~mm} \times 4.6 \mathrm{~mm}$ I.D. (Waters Corporation., Milford, MA, USA). The mobile phase was composed of solvent A $(0.05 \%$ phosphoric acid $)$ and solvent B (acetonitrile containing $0.05 \%$ phosphoric acid), and the gradients were as follows: 0-20 min, 10\%$19 \%$ B; $20-30$ min, $19 \%-29 \%$ B; $30-45$ min, $29 \%$ B; $45-50$ $\min , 29 \%-10 \% \mathrm{~B}$; and $50-60 \mathrm{~min}, 10 \% \mathrm{~B}$. The flow rate was $1.0 \mathrm{~mL} / \mathrm{min}$ at a column temperature of $25^{\circ} \mathrm{C}$. The components were detected by monitoring UV absorption at $280 \mathrm{~nm}$. Data were analyzed using
LabSolutions software (Shimadzu Co., Kyoto, Japan). Theaflavins were analyzed using the following conditions: column, ZorbaxExtend-C18, 150mm $\times 3.0$ mm I.D. (Agilent Technology, Santa Clara, CA, USA); solvent, $25 \%$ acetonitrile containing $0.05 \%$ phosphoric acid; flow rate, $1.0 \mathrm{~mL} / \mathrm{min}$; detection, $280 \mathrm{~nm}$; column temperature, $45{ }^{\circ} \mathrm{C}$. Total polyphenol content was measured byFolin-Ciocalteu method [22].

Assay of enzyme inhibitory activity: Rat intestinal acetone powder was used as the source of $\alpha$ glucosidase (EC3.2.1.20) and sucrose $\alpha$-glucosidase (sucrase) (EC3.2.1.48). The rat intestinal acetone powder was suspended in $100 \mathrm{mM}$ malate buffer, $\mathrm{pH}$ $6.9(40 \mathrm{mg} / \mathrm{mL})$, and was homogenized carefully by Ultrasonic Disruptor Model UR-200P (Tomy Seiko Co. Tokyo, Japan) at $180 \mathrm{~W}$ for $4.5 \mathrm{~min}$. After centrifugation (5600 $\times g$ for $10 \mathrm{~min}$ at $4{ }^{\circ} \mathrm{C}$ ), the resulting supernatant was used as a crude enzyme solution and diluted at a ratio of 1:2 with $100 \mathrm{mM}$ malate buffer, pH6.9, before the experiment. The experimental procedures were performed according to the method of Mai and Chuyen [23] with minor modifications. Briefly, $50 \mu \mathrm{L}$ of enzyme solution was pre-incubated with $50 \mu \mathrm{L}$ of a sample solution for $10 \mathrm{~min}$ at $37^{\circ} \mathrm{C}$. Substrate $(50 \mu \mathrm{L}$ of $2 \%$ maltose or $2 \%$ sucrose) in $100 \mathrm{mM}$ malate buffer $(\mathrm{pH}$ 6.9) was added to the mixture to start the reaction. The mixture was incubated at $37{ }^{\circ} \mathrm{C}$ for $30 \mathrm{~min}(\alpha-$ glucosidase assay) or $60 \mathrm{~min}$ (sucrase assay). After incubation, the reaction was terminated by heating with boiling water for $3 \mathrm{~min}$, and the solution was kept in an ice bath. The generated glucose was measured by the mutarotase-glucose oxidase method using Glucose Cll-test Wako according to the manufacturer's 
instructions.

The $\alpha$-amylase (EC3.2.1.1) inhibitory activity was determined using a modified version of the method of Ranilla et al. [24]. A mixture of $100 \mu \mathrm{L}$ of sample and $100 \mu \mathrm{L}$ of $20 \mathrm{mM}$ potassium phosphate buffer $(\mathrm{pH} 6.9$ with $6 \mathrm{mM}$ sodium chloride) containing $\alpha$-amylase (2 Units $/ \mathrm{mL}$ ) was incubated at $37^{\circ} \mathrm{C}$ for $10 \mathrm{~min}$. After preincubation, $100 \mu \mathrm{L}$ of $1 \%$ starch solution in the same buffer was added to each tube at timed intervals. The reaction mixture was incubated at $37^{\circ} \mathrm{C}$ for $10 \mathrm{~min}$. The reaction was stopped with $0.2 \mathrm{~mL}$ dinitrosalicylic acid color reagent followed by incubation in boiling water for $5 \mathrm{~min}$ and cooling to room temperature. The reaction mixture was then diluted with $2.5 \mathrm{~mL}$ of distilled water and the absorbance was measured at $540 \mathrm{~nm}$ using a V750 spectrophotometer (Jasco Co. Hachioji, Japan).

The $I C_{50}$, defined as the minimum concentration needed to inhibit $50 \%$ of the enzyme activity, was estimated by linear fitting. The \% inhibition was determined using the following equation:

$\%$ Inhibition $=\left(1-A_{\text {sample }} / A_{\text {control }}\right) \times 100$

Where $A_{\text {sample }}$ is the absorbance of the sample (JBT), and $A_{\text {control }}$ is the absorbance without sample.

Fractionation of JBT: JBT $(2000 \mathrm{~mL})$ was concentrated to $300 \mathrm{~mL}$ using a rotary evaporator under vacuum, and the concentrate was extracted with $3 \times 300 \mathrm{~mL}$ chloroform, ethyl acetate, and 1-butanol according to the method of Xie et al. [25]. The chloroform extract (fraction 1), ethyl acetate extract (fraction 2), 1-butanol extract (fraction 3), and remaining aqueous solution (fraction 4) were dried with a rotary evaporator under vacuum. The residue was dissolved in $100 \mathrm{~mL}$ of water.
Because $\alpha$-amylase, $\alpha$-glucosidase, and sucrase inhibitory activities were observed in fractions 2 and 3, these fractions were applied to a Sephadex LH-20 column (120 × $38 \mathrm{~mm}$ I.D.) for further separation. After washing with water to remove sugar and caffeine, the absorbed polyphenols were eluted with $30 \%$ methanol (500 mL) (fractions 2-1 and 3-1), 60\% methanol (500 $\mathrm{mL}$ ) (fractions 2-2 and 3-2), 80\% methanol (500 mL) (fractions 2-3 and 3-3), 100\% methanol (500 mL) (fractions 2-4 and 3-4) and then 60\% acetone (500 mL) (fractions 2-5 and 3-5). The solvents were removed by evaporation under reduced pressure. Fractions 2-1, 2-2, 3-1, and 3-2 were dissolved in $50 \mathrm{~mL}$ of water; fractions $2-3,2-4,2-5,3-3,3-4$, and 3-5 were dissolved in $50 \mathrm{~mL}$ of ethanol.

\section{Determination of average molecular mass by size} exclusion chromatography: The average molecular mass of components in each fraction was estimated by size exclusion chromatography according to Fujiwara et al. [26] using an HPLC system equipped with a TSK-gel a-3000 column, $300 \mathrm{~mm} \times 7.8 \mathrm{~mm}$ (Tosoh Co., Tokyo, Japan). Dimethylformamide containing $10 \mathrm{mM} \mathrm{LiCl}$ was used as the mobile phase, and elution was carried out at a flow rate of $0.3 \mathrm{~mL} / \mathrm{min}$ at a $40{ }^{\circ} \mathrm{C}$ column temperature. Fractions were monitored by UV absorption at $275 \mathrm{~nm}$. Polystyrene standards (molecular weights: 1013, 2550, 4960, 10 200, 18 500, 37 900, 96 400, and 210 000) were applied to the same column under the same chromatographic conditions to establish a calibration curve.

Thin-layer chromatography for galloyl residue detection: Tannase was dissolved in $100 \mathrm{mM}$ 2morpholinoethanesulfonic acid buffer (pH5.5) and dialyzed against the same buffer for 2 days to remove 
glucose. One $\mathrm{mL}$ of each fraction (fraction 2-3 2.34 $\mathrm{mg} / \mathrm{ml}$, fraction 2-4 $0.24 \mathrm{mg} / \mathrm{mL}$, fraction $2-50.16$ $\mathrm{mg} / \mathrm{mL}$, as a solid content) and concentrated JBT (solid content, $15.8 \mathrm{mg} / \mathrm{mL}$ ) were incubated with $1 \mathrm{~mL}$ of tannase solution ( 25 units $/ \mathrm{mL}$ ) at $30{ }^{\circ} \mathrm{C}$ for $16 \mathrm{~h}$ and then placed in a boiling water bath for 5 min to stop the reaction. Thin-layer chromatography for the detection of galloyl residues was carried out on TLC cellulose F aluminum sheets (Merck KGaA, Darmstadt, Germany) with $2 \%$ acetic acid and n-butanol/acetic acid/water $(4 / 1 / 5)$. Authentic gallic acid was used as the standard compound. The spots were colored with $0.5 \%$ iron alum.

\section{Ethics review and clinical trial registration: Two studies} were conducted to investigate the effect of JBT on postprandial blood glucose and insulin levels. These studies received the approval of the Ethics Review Committee of Takara Clinic, Medical Corporation Seishinkai on Jun 24, 2019 (Approval Number: 19061905-OS01-01-TC) and February 18, 2020 (Approval Number: 2002-1912-0S01-01-TC) and conformed to the Principles of the Declaration of Helsinki (2013) and the Ethical Guidelines for Medical Research on Human issued by the Ministry of Education, Culture, Sports, Science and Technology and Ministry of Health, Labor and Welfare, Japan. The first study lasted from July to September 2019 and the second study lasted from March to June 2020 and was registered as UMIN000037257 and UMIN000039556, respectively, in the University Hospital Medical Information Network Clinical Trials Registry, Japan.

Test beverages: JBT was poured into unlabeled bottles with plain caps. The placebo drink was prepared by dissolving $40 \mathrm{~g}$ of caramel pigment, $800 \mathrm{mg}$ of theanine,
$80 \mathrm{~mL}$ of black tea flavor, and $4 \mathrm{~mL}$ of a $50 \%$ lactic acid solution in $\mathbf{2 0}$ liters of water. The solution was sterilized at $98{ }^{\circ} \mathrm{C}$ for 30 seconds, and the mixture was stored in the same bottles as the JBT. The JBT and placebo drinks were indistinguishable in appearance, color, and aroma, and both had the following nutritional value: energy 0 $\mathrm{kcal} / \mathrm{dl}$, carbohydrates $0 \mathrm{~g} / \mathrm{dl}$, fat $0 \mathrm{~g} / \mathrm{dl}$, protein $0 \mathrm{~g} / \mathrm{dl}$, sodium chloride equivalent $0 \mathrm{~g} / \mathrm{dL}$. The JBT also contained $60 \mathrm{mg} / \mathrm{dL}$ of total polyphenols. The test beverage was labeled with an identification number and provided to the contract research organization. The identity corresponding to the identification number (JBT vs. placebo) was not provided to the contract research organization or the testing agency until after completion of the study.

Subjects: Healthy Japanese subjects over 20 years of age were recruited on the monitor recruitment site "Go Toroku" (https://www.go106.jp/). The recruitment period was June-August 2019 for the first study and February-April 2020 for the second study. The study details were disclosed to subjects before enrolment. Subjects voluntarily participated in the study and provided written informed consent. Exclusions were as follows: (i) current medical treatment for or a medical history of malignant tumor, heart failure, or myocardial infarction; (ii) a pacemaker or an implantable cardioverter defibrillator; (iii) current treatment for cardiac arrhythmia, liver disease, kidney disease, cerebrovascular disorder, rheumatism, diabetes mellitus, dyslipidemia, hypertension, or any other chronic diseases; (iv) the use of "Foods for Specified Health Uses," "Foods with Function Claims," or other functional food/beverages in daily, (v) current use of 
medications (including herbal medicines) or supplements, (vi) allergic to medicines and/or the test food-related products, (vii) pregnancy, lactation, or planning to become pregnant, (viii) enrollment in another clinical trial within three months before agreeing to participate in this trial or plans to enroll in anther clinical trial during the trial period; (ix) ineligibility as determined by the principal physician. All measured data (physical measurements, urinalysis, and blood analysis) were collected in Tokyo and imported into Access (Microsoft Office) by the staff of the contract research organization for storage.

Study design: Two studies were carried out using the same procedure. Each study was a randomized, doubleblind, placebo-controlled, crossover trial. In the first study, a screening test selected 10 subjects from 17 healthy adults. In the second study, the target number of cases was calculated with a statistical significance level $(\alpha)$ of 0.05 and a statistical detection power (1- $\beta$ ) of 0.8 based on the results of the first study. The final cohort of 36 subjects was selected from 54 applicants. These subjects were assigned to two groups by computer-generated stratified randomization, and the allocation ratio was 1:1. All participants were blind to their group assignment, and the allocation table was not opened until the data and statistical analysis was fixed. Subjects in group I consumed JBT (test 1), followed by a placebo drink (test 2); group II subjects consumed drinks in the reverse order. Each subject had at least a 7-day washout period between treatments, and the tests were conducted in the same time zone. Commercially available aseptic-pack cooked rice (Sato Food Co., Ltd. Niigata, Japan) was used as a loaded food, with the following nutritional value per serving (200 g): energy, 294 kcal; carbohydrate, $67.8 \mathrm{~g}$; protein, $4.2 \mathrm{~g}$; fat $0 \mathrm{~g}$; sodium, $0 \mathrm{mg}$. On the day of the study, subjects consumed $200 \mathrm{~mL}$ of JBT or placebo drink and $200 \mathrm{~g}$ of cooked rice within 5-10 minutes. They were required to chew the rice well, about 30 times per mouthful. Venous blood (approximately $2 \mathrm{~mL}$ ) was collected at 5 time points: baseline, and at 30, 60, 90, and 120 minutes after ingestion. Subjects were instructed (i) not to change their lifestyle and to avoid overeating or drinking from the time of giving informed consent through test completion; (ii) to avoid ingesting foods and beverages with special functions as much as possible during the test; (iii) to avoid eating and drinking except water for 6 hours before blood collection; (iv) to avoid eating or drinking anything other than test drink, loaded foods, and prescribed water from the start of blood collection to the end of the test.

The primary outcome measure was the incremental AUC of blood glucose level (iAUCglu). Secondary outcome measures were as follows: (i) maximum blood glucose level; (ii) blood glucose level; (iii) incremental AUC of the blood insulin level (iAUCins); (iv) maximum blood insulin level; (v) blood insulin level. The iAUCglu and iAUCins were calculated from the blood glucose and insulin levels measured from baseline to $120 \mathrm{~min}$ after ingestion according to the trapezoidal rule, ignoring the area below the baseline.

Analysis of blood samples: Blood glucose and serum insulin levels were analyzed by LSI Medience Co. using the enzymatic method and chemiluminescent immunoassay, respectively.

Statistical analysis: Data are presented as the mean \pm standard deviation (SD). Timing effects, carryover 
effects, and intervention effects were calculated using a generalized linear mixed model ANOVA based on the group (group I or II), nested participants, period (test 1 or 2), beverage (test or placebo drink), and study ( $1^{\text {st }}$ or $2^{\text {nd }}$ study). Statistical analysis was conducted using SPSS for Windows version 23.0 (IBM Japan Co., Ltd., Tokyo, Japan). Enzyme activities were analyzed using Student's t-test for comparison between JBT or polymer-like polyphenols and the control group. $P<.05$ based on a two-tailed test was considered statistically significant.

\section{RESULTS}

Polyphenol content: The content of catechins, theaflavins, and caffeine in JBT was as follows (mg/100 $\mathrm{mL})$ : (+)-catechin, $1.17 \pm 0.06$; (-)-catechin-3-O-gallate, $0.24 \pm 0.05 ;(-)$ gallocatechin, $0.46 \pm 0.06 ;(-)-$ gallocatechin-3-O-gallate, $0.81 \pm 0.03$; (-)-epicatechin, $2.50 \pm 0.15$; (-)-epicatechin-3-O-gallate, $4.05 \pm 0.16$; (-)epigallocatechin, $0.54 \pm 0.07$; (-)-epigallocatechin-3-Ogallate, $2.97 \pm 0.10$; theaflavine, $0.25 \pm 0$; theaflavine-3O-gallate, $0.30 \pm 0.02$; theaflavine-3'-O-gallate, $0.10 \pm 0$; theaflvaine-3,3'-di-O-gallate, $0.15 \pm 0.01$; and caffeine, $13.41 \pm 0.03$. Total polyphenol and thearubigine contents were $63.6 \pm 0.9$ and $104.1 \pm 1.6$, respectively.

Effect of JBT on digestive enzyme activity: JBT dosedependently inhibited $\alpha$-amylase, $\alpha$-glucosidase and sucrase activity, expressed as IC50 values based on dry weight (Table 1). The inhibitory effects of JBT on $\alpha-$ glucosidase and sucrase were weaker than those of salacia extract. However, JBT showed slightly stronger inhibitory activity against $\alpha$-amylase than did salacia extract. With the addition of JBT, the Michaelis constant of $\alpha$-glucosidase increased to $6.20 \pm 0.53 \mathrm{mM}$ from 4.18 $\pm 0.33 \mathrm{mM}$, and the maximal velocity decreased to 7.87 $\pm 0.24 \mu \mathrm{mol} / \mathrm{min} / \mathrm{mg}$ protein from $15.5 \pm 1.06$ $\mu \mathrm{mol} / \mathrm{min} / \mathrm{mg}$ protein. Similarly, for sucrase, the Michaelis constant increased to $34.2 \pm 1.89 \mathrm{mM}$ from $21.6 \pm 1.27 \mathrm{mM}$, and the maximal velocity decreased to $1.11 \pm 0.03 \mu \mathrm{mol} / \mathrm{min} / \mathrm{mg}$ protein from $1.61 \pm 0.03$ $\mu \mathrm{mol} / \mathrm{min} / \mathrm{mg}$ protein. These differences were statistically significant. Lineweaver-Burk plots for these enzymes showed that the inhibition by JBT was mixed non-competitive in each case. A clear result of the inhibition type could not be obtained for $\alpha$-amylase because of the interaction between the substrate and JBT. We observed that upon addition of the enzyme after pre-incubation of the inhibitor and the substrate, the inhibitory activity decreased and the IC50 value changed significantly from $1.16 \pm 0.02 \mathrm{mg} / \mathrm{mL}$ to $5.06 \pm$ $0.07 \mathrm{mg} / \mathrm{mL}$. This result suggests that the inhibitory components of JBT are bound to the substrate before binding to the enzyme. A similar phenomenon has been reported for the theaflavin digallate inhibition of $\alpha$ amylase [13]. This phenomenon was not observed in $\alpha-$ glucosidase or sucrase assays.

Table 1. IC $\mathrm{C}_{50}$ of JBT and Salacia extract for $\alpha$-glucosidase, sucrase, and $\alpha$-amylase activity

\begin{tabular}{llcc}
\hline & \multicolumn{3}{c}{ IC $_{50}$} \\
\hline & $\begin{array}{c}\alpha \text {-Gucosidase } \\
(\mu \mathrm{g} / \mathrm{mL})\end{array}$ & $\begin{array}{c}\text { Sucrase } \\
(\mathrm{mg} / \mathrm{mL})\end{array}$ & $\begin{array}{c}\alpha \text {-Amylase } \\
(\mathrm{mg} / \mathrm{mL})\end{array}$ \\
\hline JBT & $780 \pm 170$ & $1.46 \pm 0.33$ & $1.16 \pm 0.20$ \\
Salacia extract & $23.1 \pm 0.30$ & $0.05 \pm 0.02$ & $4.78 \pm 0.37$ \\
\hline
\end{tabular}

Data are presented as the mean \pm SD of three or four experiments. 


\section{Fractionation of JBT and properties of fractions}

exhibiting inhibitory activity: About $70 \%$ of the $\alpha$ -

amylase inhibitory activity was found in fraction 2

(ethyl acetate fraction) of the 4 fractions. Fraction 2

also showed the strongest inhibitory activity against $\alpha$ -

glucosidase and sucrase, followed by fraction 3 .

Fractions 1 exhibited no inhibitory activity against any

of the 3 enzymes. Fraction 2 and 3 were further

separated into 5 fractions, respectively. The $\mathrm{IC}_{50}$ and

contribution to inhibition for each fraction are shown

in Table 2. Fraction 2-3 contained about $65 \%$ of the $\alpha$ -

glucosidase inhibitory activity, with an $\mathrm{IC}_{50}$ value of

$15.2 \mu \mathrm{g} / \mathrm{mL}$. Fraction 2-3 also showed the strongest

inhibitory activity against sucrase. Fractions $2-4$ and 2-
5 contained $60 \%$ of the $\alpha$-amylase inhibitory activity,

with $\mathrm{IC}_{50}$ values of $7.99 \mu \mathrm{g} / \mathrm{mL}$ and $2.27 \mu \mathrm{g} / \mathrm{mL}$,

respectively.

Size exclusion chromatography revealed that the average molecular weight of fraction 2-3, which inhibited $\alpha$-glucosidase and sucrase, was approximately 13,000 , and that of fractions 2-4 and 2-5, which inhibited $\alpha$-amylase, were 19,000 and 30,000 , respectively (Table 3 ). The total polyphenol content expressed as grams of gallic acid equivalent was approximately $80 \%$ for fraction $2-3$, and $50 \%$ or more for fractions 2-4 and 2-5 (Table 3).

Table 2. Contribution rate and $\mathrm{IC}_{50}$ values of each fraction on $\alpha$-glucosidase, sucrase, and $\alpha$-amylase inhibitory activities

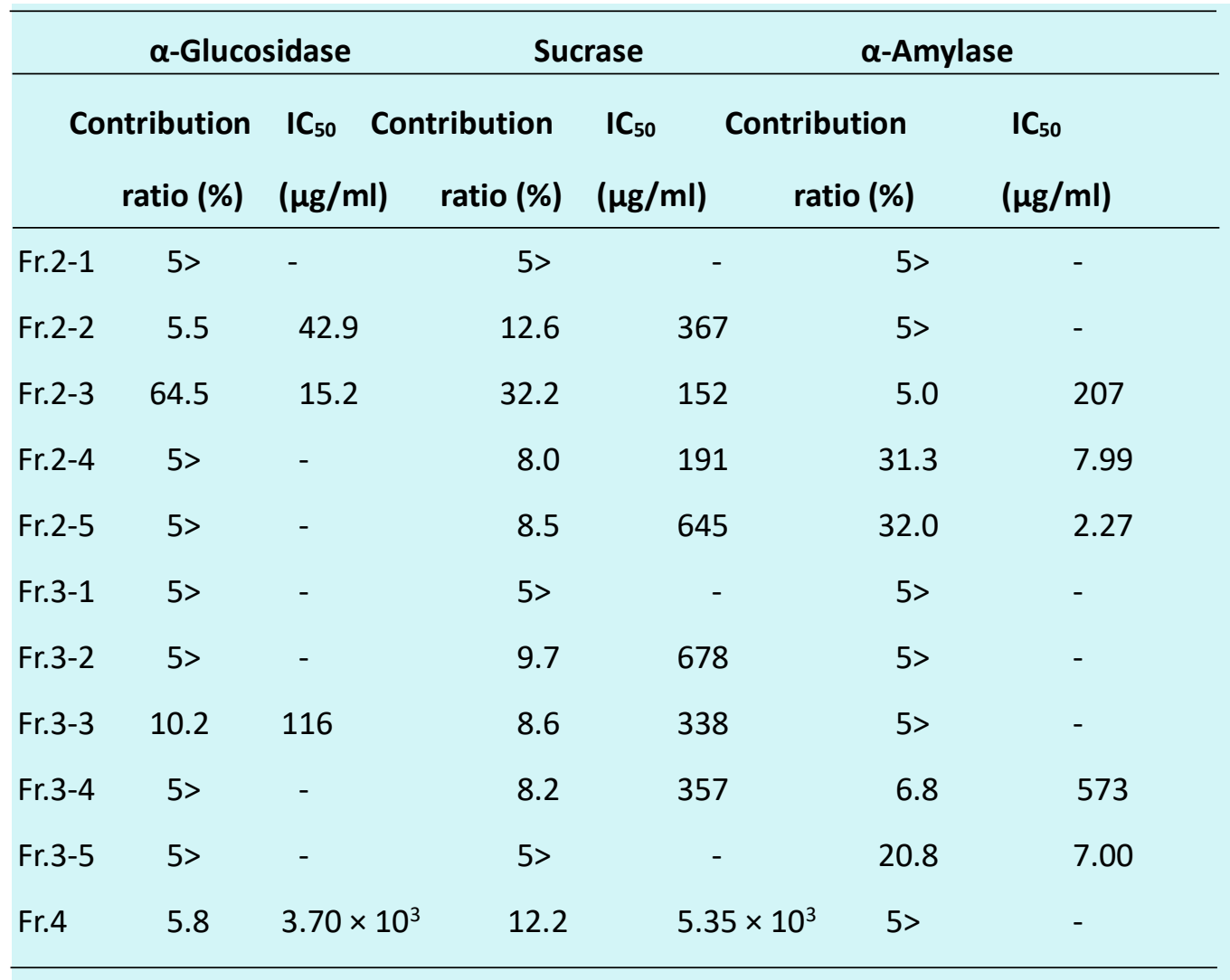


Table 3. Molecular weights and polyphenol contents of inhibitory fractions

\begin{tabular}{lccc}
\hline & Fr.2-3 & Fr.2-4 & Fr.2-5 \\
\hline Number average MW & $7.60 \times 10^{3}$ & $1.33 \times 10^{4}$ & $2.14 \times 10^{4}$ \\
Weight average MW & $1.32 \times 10^{4}$ & $1.87 \times 10^{4}$ & $2.94 \times 10^{4}$ \\
Polyphenol content (\%) & 78.5 & 55.1 & 54.0 \\
\hline
\end{tabular}

The inhibitory fractions were examined for galloyl residues in their structures. Fractions 2-3, 2-4, and 2-5 and JBT were treated with tannase, and the products were analyzed by TLC. The spots corresponded perfectly to those of the gallic acid standard ( $R f 0.68,1$ butanol/acetic acid/water (4/1/5); Rf $0.28,2 \%$ acetic acid), indicating the presence of galloyl residues. Upon tannase treatment of these fractions, the $\alpha$-amylase inhibitory activity disappeared completely, and $\alpha$ glucosidase and sucrase inhibitory activities were significantly reduced (Figure 1).

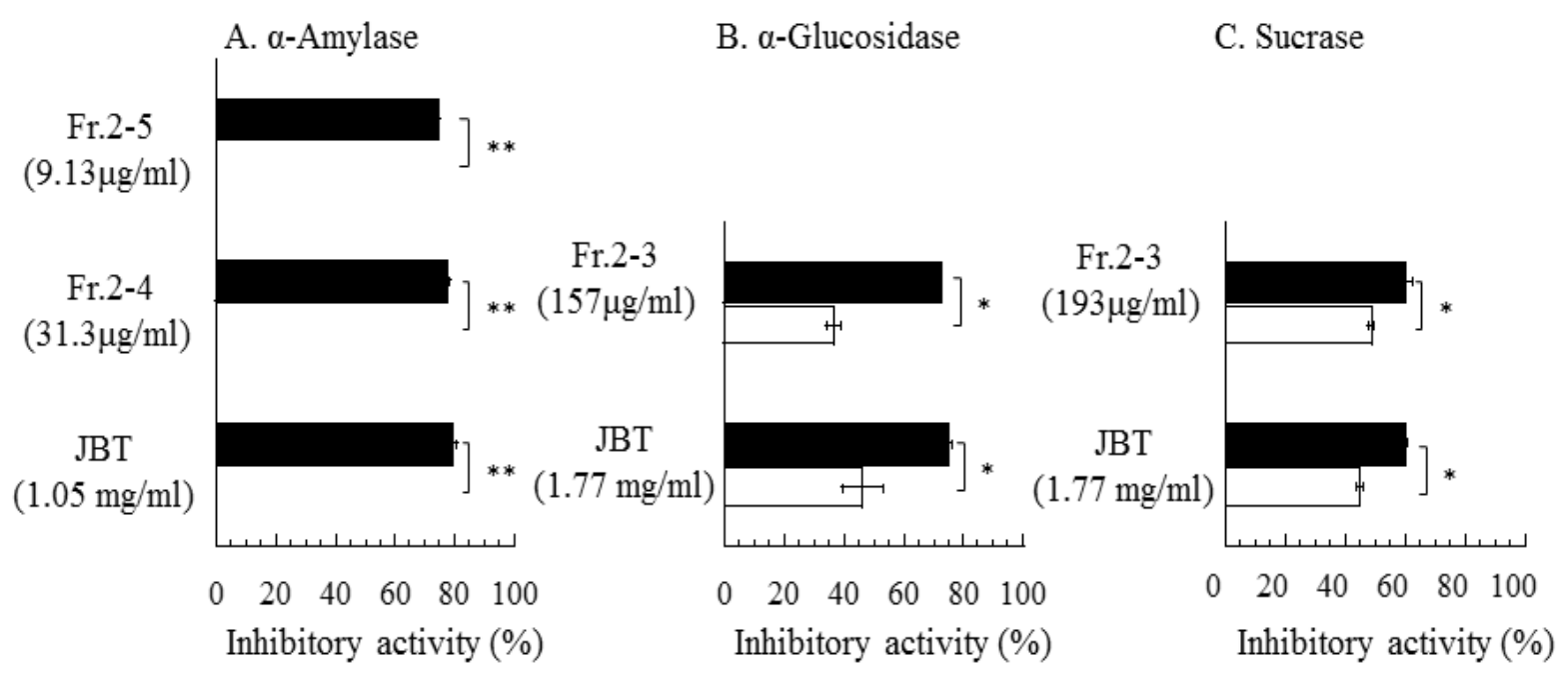

Figure 1. Effects of tannase treatment on $\alpha$-amylase, $\alpha$-glucosidase, and sucrase inhibitory activity An aliquot $(1 \mathrm{ml})$ of fraction $2-3(2.34 \mathrm{mg} / \mathrm{mL})$, fraction $2-4(0.24 \mathrm{mg} / \mathrm{mL}$, fraction $2-5(0.16 \mathrm{mg} / \mathrm{mL})$, and concentrated JBT $(15.8 \mathrm{mg} / \mathrm{mL})$ was incubated with tannase solution $(1 \mathrm{ml} ; 25$ units $/ \mathrm{mL})$ at $30{ }^{\circ} \mathrm{C}$ for $16 \mathrm{~h}$. The inhibitory activity was measured at the concentrations shown in the figure.

control; $\square$ tannase treatment; ${ }^{* *} \mathrm{P}<.01 ;{ }^{*} \mathrm{P}<.05$

Effects of JBT on postprandial blood glucose and insulin levels

Subjects: A flow chart describing the selection of subjects is shown in Figure 2. Of the total applicants ( $\mathrm{n}$ = 71) who provided informed consent and completed the pretrial test, a total of 46 subjects ( 24 females and 22 males) who met the eligibility criteria were selected and divided into two groups. No significant difference in physical exam, urine, peripheral blood, or physicochemical results was observed between groups. All subjects completed the study. No subject violated compliance or had a test food intake of less than $90 \%$. Thus, the data for all 46 subjects were analyzed. The background characteristics are shown in Table 4. 


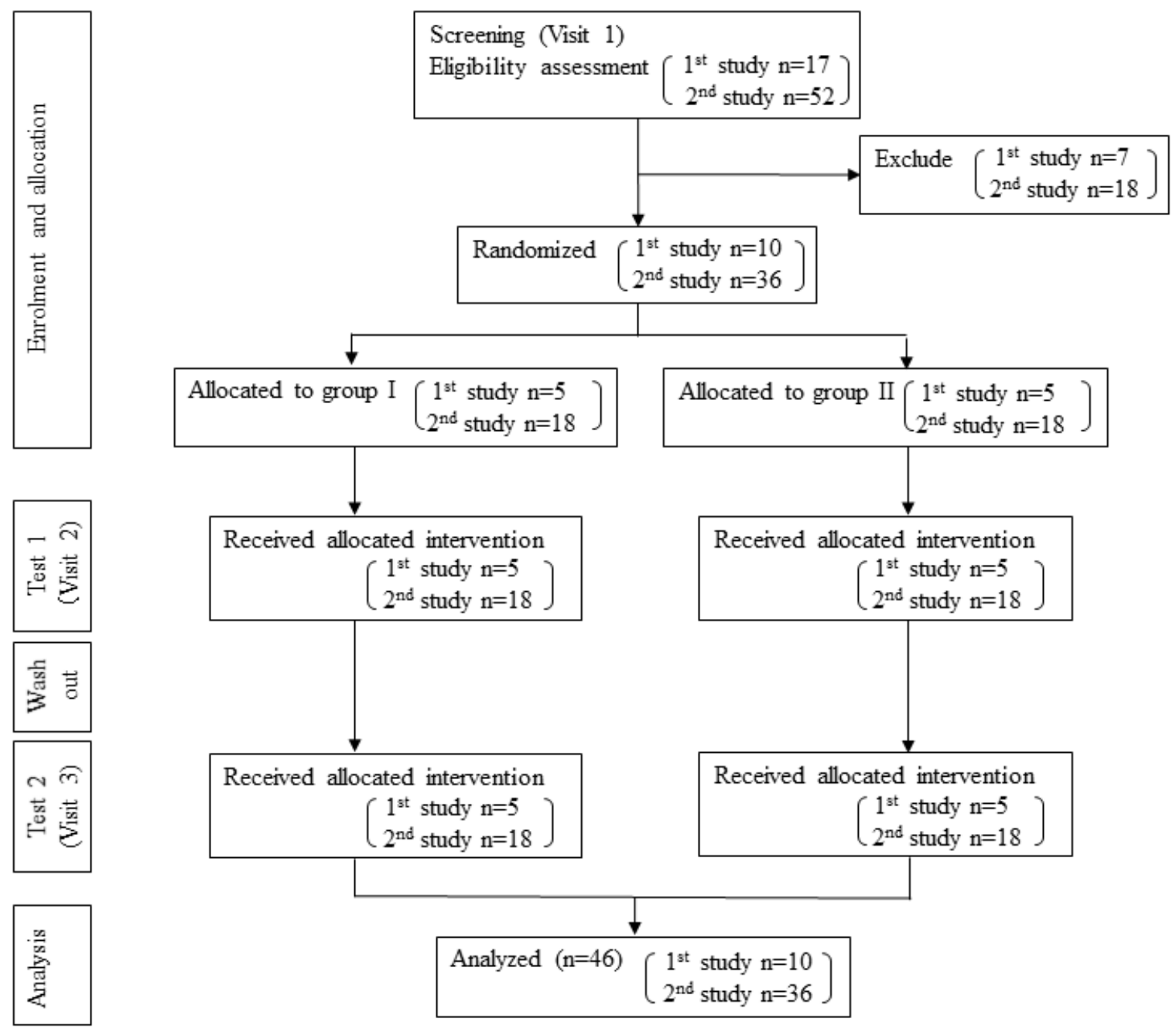

Figure 2. Study Outline

All values are expressed as the mean \pm SD.

Table 4. Background characteristics of the subjects analyzed for efficiency

\begin{tabular}{lcc}
\hline General characteristics and biological parameters & $1^{\text {st }}$ Study & $2^{\text {nd }}$ Study \\
\hline Total number, male/female & $10(4 / 6)$ & $36(18 / 18)$ \\
Age (years) & $44.8 \pm 11.0$ & $44.7 \pm 10.6$ \\
BMI (kg/m²) & $22.0 \pm 3.7$ & $22.5 \pm 3.3$ \\
Height (cm) & $162.9 \pm 7.5$ & $166.2 \pm 7.6$ \\
Body weight (kg) & $58.9 \pm 14.2$ & $62.6 \pm 12.9$ \\
Body fat (\%) & $21.1 \pm 6.2$ & $24.9 \pm 5.6$ \\
Systolic blood pressure (mmHg) & $108.2 \pm 14.7$ & $122.8 \pm 11.5$ \\
Diastolic blood pressure (mmHg) & $67.8 \pm 11.3$ & $79.1 \pm 11.0$ \\
Plus rate (beat/min) & $72.2 \pm 12.5$ & $70.3 \pm 9.5$ \\
HbA1c (NGSP) (\%) & $5.4 \pm 0.2$ & $5.3 \pm 0.2$ \\
Fasting blood glucose (mg/dl) & $80.0 \pm 6.1$ & $84.6 \pm 4.1$ \\
\hline All values are express asthe man
\end{tabular}

All values are expressed as the mean \pm SD. 


\section{Effects of JBT on blood glucose and insulin levels after}

cooked rice loading: The first study showed that the mean blood glucose level was lower after the ingestion of JBT than placebo. However, no significant difference in iAUCglu ( $P=.128)$ or iAUCins $(P=.442)$ was observed between JBT and placebo. Therefore, we conducted the second study with a larger number of subjects. In the second study, we observed a significant intervention effect of JBT for iAUCins ( $P=.021$ ) but not iAUCglu ( $P$ $=.088$ ). The mean values of iAUCglu and iAUCins were lower in JBT ingest than in placebo. Since these two studies were conducted using the same criteria and methods, we combined the data for further analysis. As shown in Table 5, a significant intervention effect of JBT was observed in iAUCglu and iAUCins $(P=.024 ; P=.014$, respectively). The baseline blood glucose and insulin levels did not differ significantly between the JBT and the placebo groups. Figure 3 shows the mean changes in blood glucose and insulin concentrations with time relative to baseline following the ingestion of cooked rice with test drinks. The blood glucose level showed a significant intervention effect by JBT $(P=.013)$ after 30 minutes, which was lower than that of the placebo group. Insulin levels were low at 90 minutes, with a significant intervention effect by JBT $(P=.009)$. JBT also had a significant intervention effect on the maximum blood glucose level $(P=.021)$ but not the maximum blood insulin level $(P=.113)$.

Table 5. The incremental area under curve of blood glucose and insulin after ingestion of cooked rice with JBT or placebo drink.

\begin{tabular}{clr}
\hline & \multicolumn{1}{l}{ JBT } & \multicolumn{2}{c}{ Placebo } \\
\hline iAUC of blood glucose $(\mathrm{mg} / \mathrm{dl} \cdot \mathrm{h})$ & $73.8 \pm 42.6^{*}$ & $85.2 \pm 44.8$ \\
$1^{\text {st }}$ study & $89.1 \pm 32.9$ & $106.2 \pm 39.5$ \\
$2^{\text {nd }}$ study & $69.5 \pm 44.3$ & $79.4 \pm 44.9$ \\
iAUC of blood insulin $(\mu \mathrm{U} / \mathrm{ml} \cdot \mathrm{h})$ & $36.9 \pm 14.1^{*}$ & $41.7 \pm 32.6$ \\
$1^{\text {st }}$ study & $31.4 \pm 13.4$ & $33.8 \pm 11.9$ \\
$2^{\text {nd }}$ study & $38.4 \pm 14.1^{*}$ & $43.9 \pm 19.2$ \\
\hline
\end{tabular}

Each value is expressed as the mean \pm SD.

*Significantly different from placebo ingestion $(P<0.05)$.

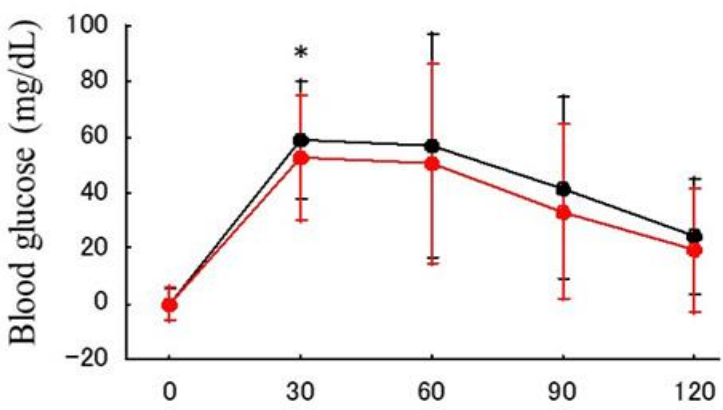

Time after ingestion $(\mathrm{min})$

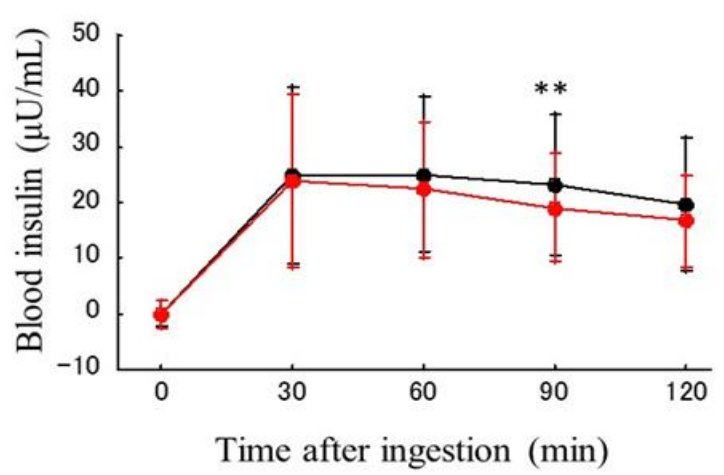

Time after ingestion $(\mathrm{min})$

Figure 3. Incremental changes in postprandial blood glucose and insulin concentrations relative to baseline in healthy subjects following consumption of cooked rice with JBT or placebo drink. Blood samples were collected before and 30, 60, 90, and 120 min after ingestion of cooked rice with JBT (empty circle) or placebo drink (solid circle). Data are presented as the mean \pm SD. Significant difference by comparison between test beverage; ${ }^{*} \mathrm{P}<.05 ;{ }^{*} \mathrm{P}<.01$. 
Adverse effects: No adverse events related to the ingestion of the test beverage occurred in this study.

\section{DISCUSSION}

In this study of the effects of JBT on postprandial blood glucose and insulin levels, we observed that black tea ingestion after cooked rice intake significantly reduced the incremental AUC of glucose and insulin compared to the placebo drinks. In vitro investigations showed that black tea dose-dependently inhibited $\alpha$ glucosidase, sucrase, and $\alpha$-amylase activity due to the action of high-molecular-weight polyphenols containing galloyl moieties. It has been reported that $150 \mathrm{mg}$ of Salacia extract containing $0.2 \mathrm{mg}$ of salacinol suppresses postprandial blood glucose elevation [27]. In this study, $200 \mathrm{ml}$ of JBT (420 mg as a dry weight) was used. Calculated from the IC50 value shown in Table 1, the $\alpha$-glucosidase inhibitory activity of this amount of JBT is about $1 / 10$ of that of $150 \mathrm{mg}$ of Salacia extract, but conversely, the inhibitory activity against $\alpha$-amylase is 10 times higher. Therefore, this inhibition of digestive enzymes may underlie the observed effect of JBT consumption on postprandial blood glucose and insulin levels.

Previous studies reported that theaflavin derivatives containing a galloyl group exhibit strong inhibitory activity against $\alpha$-amylase and $\alpha$-glucosidase $[12,13]$. Theaflavins are shown to be the primary contributors to the $\alpha$-glucosidase inhibitory activity of black tea [15]. In the present study, we observed only a small amount of theaflavins in JBT and its inhibitory fractions, contributing only $0.4 \%$ or less of the dry weight. In addition, the contribution of theaflavins to the inhibition of $\alpha$-amylase was $5 \%$ or less in fractions 2-4 and 2-5 and $12 \%$ in JBT. The inhibitory activities of theaflavins are reported to be weaker against $\alpha$ glucosidase and sucrase than $\alpha$-amylase [12, 13, 14, 28]. Consistent with this observation, we found that the theaflavin contribution to $\alpha$-glucosidase and sucrase inhibition by JBT and fraction 2-3 was less than $2 \%$. Consistent with the report by Kusano et al. [16], these results indicated that high-molecular-weight polyphenols were important contributors to the inhibition of $\alpha$-amylase, $\alpha$-glucosidase, and sucrase by JBT. Tannase treatment, which removed galloyl moieties, completely abolished the $\alpha$-amylase inhibitory activity of JBT, fraction 2-4, and fraction 2-5 and significantly decreased the $\alpha$-glucosidase and sucrase inhibitory activities of JBT and fraction 2-3. The galloyl moiety in catechin and theaflavins is important for the binding and inhibitory activity against porcine pancreatic $\alpha$-amylase [29]. Our results indicated that galloyl groups were also important in the function of high-molecular-weight polyphenols and were particularly essential for $\alpha$-amylase inhibition. While the galloyl groups were also important for $\alpha$ glucosidase and sucrase inhibition, this activity was not completely abolished as with $\alpha$-amylase.

Catechins in green tea inhibited glucose uptake by Caco-2 cells, and epicatechin gallate inhibited SGLT1, the most abundant glucose transporter in the intestine [30]. The effect of JBT on glucose uptake was not investigated in this study. However, Satoh et al. [31] reported that black tea extract does not inhibit SGLT1 or GLUT2 in Caco-2 cells, and the catechin content of JBT was similar to that of the black tea used in their investigation. The total catechins in JBT are about $1 / 6$ to $1 / 3$ that of commercial bottled green tea, which may not be enough to inhibit glucose transporters such as SGLT1.

JBT significantly decreased iAUCglu and iAUCins after cooked rice intake compared with the placebo drink. Glucose is the most important insulin secretagogue, and insulin is released into the blood upon stimulation of glucose uptake into pancreatic beta cells [32]. Therefore, the observed decrease in iAUCglu 
and iAUCins likely resulted from the suppression of glucose uptake by JBT. We infer that the inhibition of $\alpha$ glucosidase and $\alpha$-amylase activities by the highmolecular-weight polyphenols contained in JBT suppressed the release of glucose in the digestive tract, resulting in lower postprandial blood glucose and insulin levels.

Caffeine ingestion is reported to increase insulin levels during glucose tolerance testing [33]. Bryans et al. [20] reported that the ingestion of caffeine-containing instant black tea with glucose caused elevated insulin levels. However, the elevation in insulin following caffeine ingestion was not mirrored by an increase in insulin at the same time point after tea ingestion, suggesting a difference in bioavailability between different sources of caffeine. In our study, the ingested JTB $(200 \mathrm{~mL}$ ) contained $26.82 \mathrm{mg}$ of caffeine, which is about half that of the study by Bryans et al. This difference in caffeine intake may explain the different results. In addition, differences in the polyphenol content of the black tea and its binding properties to caffeine may affect the bioavailability of caffeine and the consequent insulin level.

The suppression of postprandial blood glucose and insulin levels by JBT may be attributable to the inhibition of digestive enzymes by high-molecularweight polyphenols containing galloyl groups. However, clarification of the effects of caffeine, catechins, and polyphenols on insulin secretion and glucose transporter inhibition requires further study. The present results suggest that drinking JBT in amounts easily consumed with food containing starch is beneficial for glycemic control and may help reduce the risk of diabetes in healthy people. In this study, we investigated the effect of a single dose on postprandial blood glucose levels. The effects of long-term intake need to be investigated in future.

\section{CONCLUSION}

JBT inhibits the breakdown of starch by $\alpha$-amylase and $\alpha$-glucosidase. We determined that the components of JBT primarily responsible for this inhibition are highmolecular-weight polyphenols containing galloyl groups. This result suggests that the observed JBT-induced decrease in blood glucose and insulin levels after rice consumption resulted from the inhibition of these starch degradation enzymes.

Abbreviations: AUC: Area under the curve, iAUCglu: Incremental area under the curve of blood glucose level, iAUCins: Incremental area under the curve of blood insulin, JBT: Java black tea, SD: Standard deviation

Competing interests: The human trial was supported by funding from Otsuka Foods Co. Ltd. and was outsourced to ORTHOMEDICO Inc. Although Y.I., H. W. and M. K. are employees of Otsuka Foods Co., Ltd., these authors are not involved in the interpretation of the results of human experiments, and the independence of the investigators and the study is ensured. The other authors have no conflict of interests to declare.

Author contribution: Y. I. designed the study. Y. I. and H. W. carried out the enzyme inhibitory experiments. M.K. analyzed the components of the test beverage and fractions. T.T conducted the human trial. S. I. wrote the human trial protocol and managed the trial. Y. I. wrote and revised the manuscript. All the authors have read and approved the final version of the manuscript.

Acknowledgments: We would like to thank the staff of ORTHOMEDICO Inc. for their cooperation and advice in the human test. The production of the test beverage was supported by Sayaka Yono, Tetsushi Kodama of Biwako Research Institute and the stuff of Shiga Factory of Otsuka Foods. Co. Ltd. We would also like to thank Enago (www.enago.jp) for the English language review. 


\section{REFERENCES}

1. International Diabetes Federation, Diabetes Atlas $9^{\text {th }}$ edition, [https://www.diabetesatlas.org/en/sections/demographi c-and-geographic-outline.html] Retrieved Dec. 30, 2019.

2. Bonara, E, Muggeo, M: Postprandial blood glucose as a risk factor for cardiovascular disease in type II diabetes; the epidemiological evidence. Diabetologia, 2001, 44 2107-14.

3. Celliero, A: The glucose triad and its role in comprehensive glycemic control: Current status, future management. Int J Clin Prac, 2010, 64(12): 1705-11.

4. Cheng, A, Josse, R: Intestinal absorption inhibitors for type 2 diabetes mellitus: Prevent and treatment. Drug Discov Today, 2004, 1(2): 201-6.

5. Puls, W, Keup, U, Krause, H, Thomas, G, aHoffmeister, F: Glucose inhibition: A new approach to the treatment of diabetes, obesity and hyperlipoproteinemia. Naturwissenschaften, 1977, 64: 536-7.

6. Kumar, S, Narwal, S, Kumar, V, Prakash, O: $\alpha$-Glucosidase inhibitors from plants: A natural approach to treat diabetes. Pharmacogn Rev, 2011, 5(9): 19-9.

7. Kwon, Y, Apostolidis, E, Shetty, K: Inhibition potential of wine and tea against $\alpha$-amylase and $\alpha$-glucosidase for management of hyperglycemia linked to type 2 diabetes. J Food Biochem, 2006, 32: 15-31.

8. Sun, L, Chen, W, Meng, Y, Yang, X, Yuan, L, Guo, Y: Interactions between polyphenols in thinned young apples and porcine pancreatic $\alpha$-amylase: Inhibition, detailed kinetics and fluorescence quenching. Food Chem, 2016, 208(October): 51-60.

9. McDougall, G, Shpiro, F, Dobson, P, Smith, P, Blake, A, Stewart, D: Different polyphenolic components of soft fruits inhibit $\alpha$-amylase and $\alpha$-glucosidase. J Agric Food Chem, 2005, 53(7): 2760-66.

10. Hararika, M, Chakravarty, SK, Mahanta, PK: Studies on thearubigin pigments in black tea manufacturing systems. J Sci Food Agric, 1984, 35(11): 1208-18.

11 Striegel, L, Kang, B, Pilkenton, SJ, Rychlik, M, Apostolides, E: Effect of black tea and black tea pomace polyphenols on $\alpha$-glucosidase and $\alpha$-amylase inhibition, relevant to type 2 diabetes prevention. Front Nutr, 2015, 2: 1-6.

12. Honda, M, Hara, Y: Inhibition of rat small intestinal sucrase and $\alpha$-glucosidase activities by tea polyphenols. Biosci Biotech Biochem, 1993, 57(1): 123-4.

13. Hara, $Y$, Honda, $M$ : The inhibition of $\alpha$-amylase by tea polyphenols. Agric Biol Chem, 1990, 54(8): 1939-45.

14. Matsui, T, Tanaka, T, Tamura, S, Toshima, A, Tamaya, K, Miyata, Y, Tanaka, K, Matsumoto, K: $\alpha$-Glucosidase inhibitory profile of catechins and theaflavins. J Agric Food Chem, 2007, 55(1): 99-105.

15. Uchida, N, Yoshimoto, N, Nakamura, A, takagi, A, Honda,
K, Moto, M, Katsuraya, K, Hashizume, N: Difference of inhibition effect of $\alpha$-glucosidase by tealeaves species and extraction condition and effect of black tea on postprandial blood glucose level elevation in ICR mice. J Anal Bio-Sci, 2013, 36(2): 193-202.

16. Kusano, R, Andou, H, Fujieda, M, Matsuo, Y, Kouno, I: Polymer-like polyphenols of black tea and their lipase and amylase inhibitory activities. Chem Pharm Bull, 2008, 56(3): 266-272.

17. Abeywickrama, KRW, Ratnasooriya, WD, Amarakoon, AMT: Oral hyperglycaemic, antihyperglycaemic and antidiabetic activities of Sri Lankan broken orange pekoe fannings (BOPF) grade black tea (Camellia sinensis L.) in rats. J Ethnopharmcol, 2011, 135: 278-286.

18. Odegaard, AO, Pereira, MA, Koh, WP, Arakawa, K, Lee, HP, Yu, MC: Coffee, tea, and incident type 2 diabetes: The Singapore Chinese Health Study. Am J Clin Nutr, 2008, 88:979-985

19. Zhong, L, Furne, JK, Levitt, MD: An extract of black, green, and mulberry teas causes malabsorption of carbohydrate but not of triacylglycerol in healthy volunteers. Am J Clin Nutr, 2006, 84: 551-5.

20. Bryans, JA, Judd, PA, Ellis, PR: The effect of consuming instant black tea on postprandial plasma glucose and insulin concentrations in healthy humans. J Am Coll Nutr, 2007, 26(5): 471-7.

21. Butacnum, A, Chongsuwat, $R$, Bumrungpert, $A$ : Black tea consumption improves postprandial glycemic control in normal and pre-diabetic subjects: A randomized, doubleblind, placebo-controlled crossover study. Asia Pac J Clin Nutr, 2017, 26(1): 59-64.

22. Determination of substances characteristic of green and black tea -- Part 1: Content of total polyphenols in tea -Colorimetric method using Folin-Ciocalteu reagent, ISO 14502-1, 2005.

23. Mai, TT, Chuyen, NV: Anti-hyperglycemic activity of an aqueous extract from flower buds of Cleistocalyx operculatus (Roxb.) Merr and Perry. Biosci Biothech Biochem, 2007, 71(1): 69-76.

24. Ranilla, LG, Kwon, YI, Apostolidis, E, Shetty, K: Phenolic compounds, antioxidant activity and in vitro inhibitory potential against key enzymes relevant for hyperglycemia and hypertension of commonly used medical plants, herbs and spices in Latin America. Bioresour Thechnol, 2010, 101: 4676-89.

25. Xie, B, Shi, H, Chen, Q, Ho,CT: Antioxidant properties of fractions and polyphenol constituents from green, oolong and black teas. Proc Natl Sci Counc Repub China B, 1993, 17(2): 77-84.

26. Fujiwara, T, Nakagawa-Izumi A, Ozawa, T, Numata, O: High-molecular-weight polyphenols from oolong tea and black tea: Purification, some properties, and role in increasing mitochondrial membrane potential. Biosci Biotech 
Biochem, 2007, 71(3): 711-9.

27. Beppu, H, Shikano, M, Fujita, K, Itani, Y, Hamayasu, K, Kishino, E, Ito, T, Ozaki, S, Shinpo, K, Sonoda, S, Higashiguchi, $\mathrm{T}$ : Effect of single and 3-month repeated ingestion of a Kotahalahim granule preparation containing Kotala himbutu extract on the glucose metabolism of humans. J Foodingred, 2005, 8(2): 105-117.

28. Koh, LW, Wong, LL, Loo, YY, Kasapis, S, Hung, D: Evaluation of different teas against starch digestibility by mammalian glycosidases. J Agric Food Chem, 2010, 58:148-54.

29. Sun, L, Gidley, MJ, Warren, FJ: The mechanism of interactions between tea polyphenols and porcine pancreatic alpha-amylase: Analysis by inhibition kinetics, fluorescence quenching, differential scanning calorimetry and isothermal titration calorimetry. Mol Nutr Food Res, 2017, 61(10): 1700324

30. Shimizu, M, Kobayashi, Y, Suzuki, M, Satsu, H, Miyamoto, $\mathrm{Y}$ : Regulation of intestinal glucose transport by tea catechins. BioFactors, 2000, 13(1-4): 61-5.

31. Satoh, T, Igarashi, M, Yamada, S, Takahashi, N, Watanabe, $\mathrm{K}$ : Inhibitory effect of black tea and its combination with acarbose on small intestinal $\alpha$-glucosidase activity. J Ethnopharmacol, 2015, 161: 147-55.

32. Amano, E, Fujimoto, S: $\beta$ cell dysfunction diabeates. Tonyobyo, 2016, 59(5): 322-5 (in Japanese).

33. Graham, T, Sathasivam, P, Rowland, M, Maruko, N, Greer, $F$, Battram D: Caffeine ingestion elevates plasma insulin response in humans during an oral glucose tolerance test. Can J Physiol Pharmacol, 2001, 79(7): 559-5. 